\title{
MULTIRESOLUTION MOMENT FILTERS
}

\author{
Michael Sühling, Muthuvel Arigovindan, Patrick Hunziker*, and Michael Unser \\ Swiss Federal Institute of Technology Lausanne \\ Biomedical Imaging Group, STI/IOA \\ CH-1015 Lausanne, Switzerland \\ e-mail: michael.suehling@epfl.ch, muthuvel.arigovindan@epfl.ch, michael.unser@epfl.ch
}

\begin{abstract}
We define multi-scale moments that are estimated locally by analyzing the image through a sliding window at multiple scales. When the analysis window satisfies a two-scale relation, we prove that these moments can be computed very efficiently using a multiresolution wavelet-like algorithm. We also show that B-spline windows are best suited for this kind of analysis because, in addition to being refinable, they are positive, symmetric and very nearly isotropic.

We present two applications of our method. The first is a feature extraction method for detecting strands of DNA in noisy cryoelectron-micrographs. The second is an extension of the LucasKanade optical flow algorithm that assumes a local affine model of the motion field. The results obtained in both cases are very promising.
\end{abstract}

\section{INTRODUCTION}

Global moments and their invariants are widely used in many areas of image analysis, including pattern recognition [1], image reconstruction [2], and shape identification [3]. Some authors have also applied moments in a local fashion for image segmentation [4] and direction-based interpolation [5]. The idea there was to compute moments locally over some square region of interest which is moved over the image; the observation windows may be overlapping or not depending on the application.

In this paper, we are extending the notion of local moments by introducing two refinements: weighting and multiresolution. The idea of weighting is motivated by the observation that the square window that has been used so far is rather anisotropic. Indeed, if the goal is to design a "rotation-invariant" algorithm, it makes good sense to apply an isotropic window with a radial weighting that decreases away from the center. Multiresolution is a feature that is highly desirable for designing image processing algorithms that have some degree of adaptability. The down-side, of course, is that these refinements can be computationally very expensive, especially when the size of the window is large. In Section 2, we will show how to overcome these limitations by proposing a multiresolution wavelet-like algorithm to compute multi-scale local moments of different orders with a dyadic scale progression. In particular, we will consider B-spline weighting windows, which

${ }^{*}$ P. Hunziker is with the Kantonsspital Basel, Petersgraben 5, CH-4031 Basel, e-mail: PHunziker@uhbs.ch.

This work was supported in part by the Swiss National Science Foundation under grant 3200-059517.99 and the Swiss Heart Foundation. become wider and more and more Gaussian-like-also meaning isotropic - as the degree of the spline increases.

We believe that these multi-scale local moments should be useful tools for devising new algorithms based on what we call a "sliding window" formulation of a problem. The basic assumption for such an approach is that the spatially-varying feature (or parameter) that one is estimating is approximately constant within the observation window. The unknown parameter is then estimated from the available information in the window (which often requires the evaluation of moments). Finally, the output value is attributed to the spatial location corresponding to the center of the window. This is a simple, yet powerful paradigm that can be made most effective by working at the appropriate scale (multiresolution strategy). We will illustrate these ideas in Section 3 by presenting two such local-moment-based algorithms:

- a new method for detecting DNA strands in very noisy cryoelectron-micrographs,

- a multiresolution extension of the Lucas-Kanade optical flow algorithm [6], which uses a more refined local-affine model for the motion.

These methods are fast thanks to our wavelet-like implementation. The experimental results obtained in both cases are very encouraging.

\section{THEORY}

In this section, we will define weighted local moments and show how they can be computed efficiently in a multiresolution framework.

\subsection{Weighted Local Moments}

Let $w$ be a positive window function of support $\Omega$. Given some integer $p$, we define

$$
w_{p}(x)=x^{p} w(x) .
$$

For a continuously-defined function $f(x)$, we define the local moment of order $p$, scale $j \in \mathbb{Z}$, and location $x_{0}$ as

$$
m_{p}^{(j)}\left(x_{0}\right)=\int_{\Omega} w_{p}\left(\frac{x-x_{0}}{2^{j}}\right) f(x) d x .
$$

Note that the window function is dilated by a factor $2^{j}$ and is centered at $x_{0}$, which is typically an integer in the discrete case. 


\subsection{Two-Scale Equation}

Computing local moments at coarser scales becomes very expensive due to the increasing size of the window function. However, multiresolution pyramids of local moments can be computed efficiently as in wavelet-like algorithms, provided that the window function satisfies a two-scale equation.

Theorem 1 (Two-Scale Equation) Let $w$ be a function which satisfies the two-scale equation

$$
w\left(\frac{x}{2}\right)=\sum_{l} h(l) w(x-l),
$$

for some given filter $h$. Then, $w_{p}$ satisfies the two-scale equation

$$
w_{p}\left(\frac{x}{2}\right)=\sum_{k=0}^{p}\left(h_{p, k} * w_{k}\right)(x),
$$

with filters $h_{p, k}, k=0, \ldots, p$, given by

$$
h_{p, k}(l)=\frac{1}{2^{p}}\left(\begin{array}{l}
p \\
k
\end{array}\right) l^{p-k} h(l) .
$$

Note that $h_{0,0}=h$.

\subsection{Efficient Multi-Scale Implementation}

Theorem 1 can be used to derive fast algorithms for computing the local moments $m_{p}^{(j)}$ in (2) for scales $j=j_{0}, \ldots, j_{1}$ and orders $p=0, \ldots, P$. To initialize the procedure, we compute the inner products on the finest scale $j_{0}$ by using (2). Due to Theorem 1 , the coefficients on the subsequent coarser scales can be determined recursively as

$$
m_{p}^{(j+1)}(n)=\sum_{k=0}^{p} \sum_{l} h_{p, k}(l) m_{k}^{(j)}\left(n+2^{j} l\right) .
$$

Equation (6) is a multi-channel extension of the "à trous" algorithm, which is frequently used for computing overcomplete wavelet transforms [7]. The method is easily modified for computing local moments in a sub-sampled, wavelet-like pyramid. The recursion equation (6) then simplifies to a Mallat-like algorithm (cf. [7]).

If we assume that the length of the discretized window $w$ is $N$, the direct calculation of (2) is of complexity $2^{j} N$ for scale $j$ and any order $p$. In contrast, the complexity of $(6)$ is $(p+1) N$ for each scale and for order $p$. Obviously, the use of the recursion equation pays off, if $2^{j}>(p+1)$.

\subsection{Multiple Dimensions}

Equation (6) can be applied to multiple dimensions in a straightforward way by using tensor products. In the $2 \mathrm{D}$ case, we define window functions and corresponding moments of order $(p+q)$ as

$$
w_{p, q}(x, y)=x^{p} y^{q} w(x) w(y)
$$

and

$$
m_{p, q}^{(j)}\left(x_{0}, y_{0}\right)=\int_{\Omega} w_{p, q}\left(\frac{x-x_{0}}{2^{j}}, \frac{y-y_{0}}{2^{j}}\right) f(x, y) d x d y .
$$

For an efficient computation of $m_{p, q}^{(j)}$, equation (6) can be applied separately in each dimension.

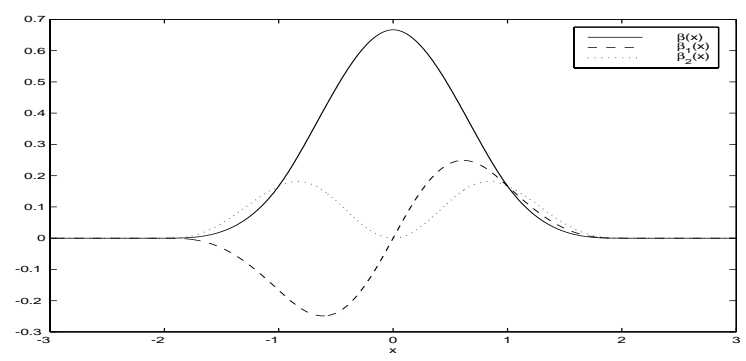

Fig. 1. Cubic B-spline $\beta^{3}$ and its first two moment filters $\beta_{1}^{3}$ and $\beta_{2}^{3}$.

\subsection{Choice of Window Function}

Among the window functions, a case of special interest is $w=\beta^{n}$, where $\beta^{n}$ is the symmetrical B-spline of degree $n \in \mathbb{N}$ [8]. It satisfies a two-scale equation and the shape of the window can be modified by choosing B-splines of different degrees. Additionally, B-splines rapidly converge to Gaussians when their degree increases. This ensures isotropy of the window in multiple dimensions. The cubic B-spline $(n=3), \beta^{3}$, and its two first moments $\beta_{1}^{3}$ and $\beta_{2}^{3}$ are plotted in Figure 1. The corresponding two-scale filters $h_{p, k}$ up to order $p=2$ are given in Table 1 .

Table 1. Two-scale filters $h_{p, k}$ up to order $p=2$ for $\beta^{3}$.

\begin{tabular}{|c||c|c|c|c|c|}
\hline$l$ & -2 & -1 & 0 & 1 & 2 \\
\hline \hline$h_{0,0}(l)$ & $\frac{1}{8}$ & $\frac{4}{8}$ & $\frac{6}{8}$ & $\frac{4}{8}$ & $\frac{1}{8}$ \\
\hline$h_{1,0}(l)$ & $-\frac{1}{8}$ & $-\frac{2}{8}$ & 0 & $\frac{2}{8}$ & $\frac{1}{8}$ \\
\hline$h_{1,1}(l)$ & $\frac{1}{16}$ & $\frac{4}{16}$ & $\frac{6}{16}$ & $\frac{4}{16}$ & $\frac{1}{16}$ \\
\hline$h_{2,0}(l)$ & $\frac{1}{8}$ & $\frac{1}{8}$ & 0 & $\frac{1}{8}$ & $\frac{1}{8}$ \\
\hline$h_{2,1}(l)$ & $-\frac{1}{8}$ & $-\frac{2}{8}$ & 0 & $\frac{2}{8}$ & $\frac{1}{8}$ \\
\hline$h_{2,2}(l)$ & $\frac{1}{32}$ & $\frac{4}{32}$ & $\frac{6}{32}$ & $\frac{4}{32}$ & $\frac{1}{32}$ \\
\hline
\end{tabular}

\section{APPLICATIONS}

The fast algorithm presented above is applicable to a whole variety of image analysis problems, such as image segmentation, pattern detection, and optical flow estimation, for which local solutions over sliding windows have been proposed. These approaches can also be extended by applying a multiresolution strategy which provides adaptability while also reducing computational cost. Here, we will illustrate the concept by presenting new local-momentbased algorithms for two specific tasks: (1) the detection of DNA strands, and (2) the estimation of motion fields using a local affine model.

\subsection{Detection of DNA Strands}

The structure of DNA molecules can be visualized by cryo-electronmicroscopy (CEM) [9]. Because of the physical process involved, the resulting images have very low contrast to avoid destruction of the specimen (cf. Figure 2). Biologists are highly interested in an automatic detection of the thin strands of DNA, but the task is extremely challenging because of the poor signal-to-noise ratio (near 
OdB). Prior to coming up with our new moment method, we had tried many other techniques without obtaining satisfactory results. Since local moments are integral-based features, they are robust against noise and therefore well suited to analyzing such objects which have a marked elongated structure.

The moments $m_{p, q}$ have well defined geometric interpretations. The coordinates of the local centroid are given by

$$
\bar{x}=m_{1,0} / m_{0,0} \quad \text { and } \quad \bar{y}=m_{0,1} / m_{0,0} .
$$

The so-called central moments [1] can be expressed in terms of ordinary moments $m_{p, q}$ and the coordinates of the centroid. The three central moments of second order can be used to build an inertia matrix $\mathbf{J}$. The local orientation of the object is given by the eigenvector corresponding to the minimal eigenvalue of $\mathbf{J}$. The eccentricity $\varepsilon=\left(\lambda_{1}-\lambda_{2}\right)^{2} /\left(\lambda_{1}+\lambda_{2}\right)^{2}$, where $\lambda_{1}$ and $\lambda_{2}$ are the eigenvalues of $\mathbf{J}$, takes values between 0 and 1 , indicating whether the local object is elongated or not.

The DNA strands are detected at multiple scales $j_{0} \leq j \leq j_{1}$, where $j_{0}$ and $j_{1}$ are the finest and coarsest scale at which relevant structures are expected. At each image pixel $\left(n_{x}, n_{y}\right)$ we compute the local moments $m_{p, q}^{(j)}$ for $(p+q) \leq 2$. From these we derive the local orientations and eccentricities $\varepsilon^{(j)}$. To decide whether or not a local object is part of a DNA strand, we compute the figure of merit

$$
\gamma^{(j)}=\varepsilon^{(j)} e^{-\left(\bar{x}^{2}+\bar{y}^{2}\right) /\left(2^{(2 j+1)} \sigma^{2}\right)} .
$$

The second factor in (10) assigns more weight to cases where the local centroid $(\bar{x}, \bar{y})$ is close to the center of the local window. The parameter $\sigma$ controls the range of the centroid around the window origin to be accepted. The multi-scale approach also helps us to detect cases where the local structure is located symmetrically at the periphery of the window function. To avoid these cases, the figure of merit $\gamma^{(j)}$ is set to zero, if $m_{0,0}^{(j-1)}<m_{0,0}^{(j)}$. This means that the local mean of the gray values at the next finer scale has to be greater than the local mean at the current scale.

The figure of merit (10) will be maximal at a scale that approximately matches the size of the DNA strand to detect. Therefore, we integrate the figures of merit at different scales to obtain a final estimate for the goodness of local fit by $\gamma=\max _{j_{0} \leq j \leq j_{1}} \gamma^{(j)}$.

\section{Numerical Results}

As can be seen in Figure 2(a), the intensity in CEM-images may vary globally. Therefore, the original images were first normalized in a pre-processing step. We used moments of order zero at scale $j=2$ for local background subtraction. Then we computed for each pixel the figure of merit $\gamma$ as described above. The figures of merit were then thresholded to suppress values that correspond to non-significant structures. The final figures of merit are visualized in Figure 2(b) in form of a needle diagram. The length of the needles is proportional to the size of the figure of merit at each pixel. The direction of the needles corresponds to the local orientation of the object. We see that the two DNA strands contained in the image together with their local orientation were clearly detected. Failures due to the high noise content in the image are very sparse.

\subsection{Optical Flow Estimation}

Let $I(x, y, t)$ denote the intensity of pixels at location $\mathbf{r}=(x, y)$ and time $t$ in an image sequence. Gradient-based optical flow estimation relies on the assumption that the intensity of a particular

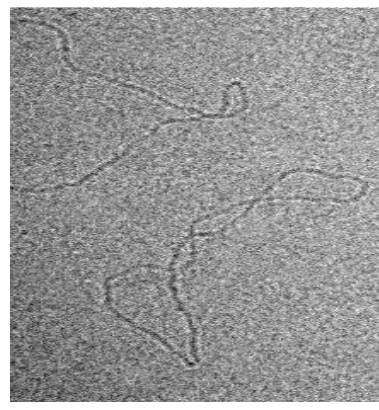

(a) Original CEM-Image

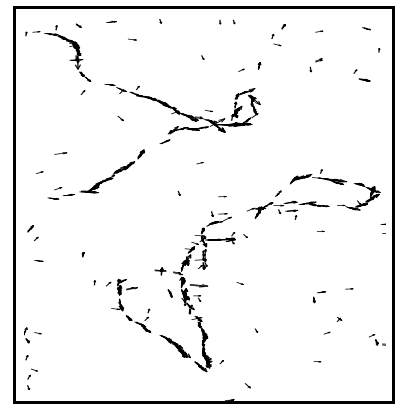

(b) Estimated Local Orientation
Fig. 2. A CEM-image and detected DNA strands

point in a moving pattern does not change with time. The constant intensity assumption can be expressed as [10]

$$
I_{x}(\mathbf{r}, t) u(\mathbf{r}, t)+I_{y}(\mathbf{r}, t) v(\mathbf{r}, t)+I_{t}(\mathbf{r}, t)=0 .
$$

$I_{x}, I_{y}$ and $I_{t}$ denote the spatial and temporal derivatives of the image intensity. The velocities $u$ and $v$ are, respectively, the $x$ and $y$-components of the optical flow we wish to estimate.

\section{Local Affine Motion}

A very popular optical flow algorithm is the Lucas-Kanade method [6], which estimates the motion locally, assuming that motion is constant within a window of support $\Omega$. In order to account for more complex motions, such as rotation, divergence, and shear, we extend this approach to a local affine model for the motion. If $\left(x_{0}, y_{0}\right)$ denotes the center of the local window, this model is defined as

$$
\begin{aligned}
& u(x, y)=u_{0}+u_{x}\left(x-x_{0}\right)+u_{y}\left(y-y_{0}\right) \\
& v(x, y)=v_{0}+v_{x}\left(x-x_{0}\right)+v_{y}\left(y-y_{0}\right) .
\end{aligned}
$$

The parameters $u_{0}$ and $v_{0}$ correspond to the motion at the window center and $u_{x}, u_{y}, v_{x}$, and $v_{y}$ are, respectively, the first order spatial derivatives of $u$ and $v$. The local motion components can be estimated by minimizing the least-squares criterion

$$
\int_{\Omega} w(x, y)\left(I_{x} u+I_{y} v+I_{t}\right)^{2} d x d y .
$$

The symmetric window function $w$ gives more weight to constraints at the center of the local region than to those at the periphery. By differentiating (13) with respect to each of the six unknown parameters, we obtain a linear system $\mathbf{A v}=\mathbf{b}$ in terms of local moments of orders zero to two of the spatial and temporal derivatives of $I$.

It is obviously difficult to estimate large motions at fine scales. A way around this problem is to apply a coarse-to-fine strategy. At each scale $j$ we compute the local moments on a grid which is sub-sampled by $2^{j}$ in each dimension. Due to this sub-sampling, equation (6) reduces to a simple convolution.

The motion estimates are cascaded through each resolution level as initial estimates and are then refined if possible. This strategy is sketched in the following algorithm. 


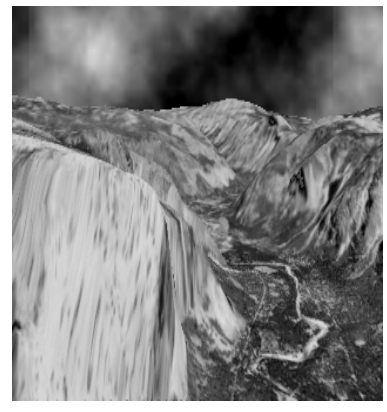

(a) Frame

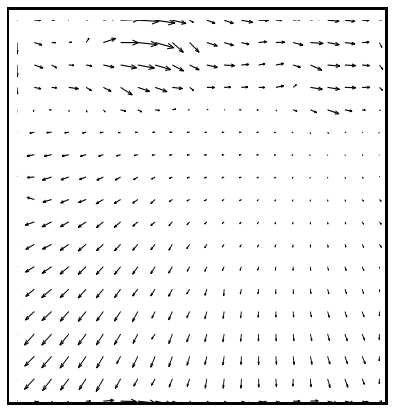

(b) Estimated Motion

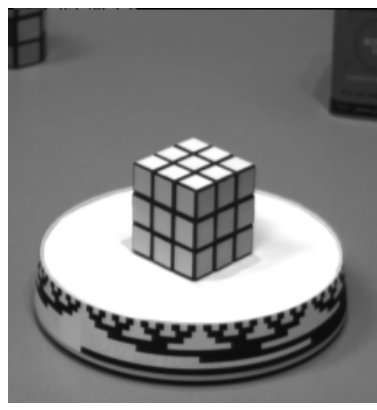

(a) Frame

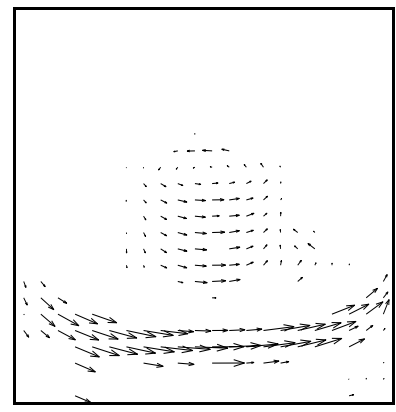

(b) Estimated Motion
Fig. 3. One frame of the Yosemite sequence and its corresponding estimated motion field.

Input: Multi-scale pyramids of local moments of the spatial and temporal derivatives of $I$.

Step 1: Start at coarsest scale: $j:=j_{\max }$

Step 2: for each grid position $2^{j} \mathbf{n}$ do

Compose local linear system $\mathbf{A v}=\mathbf{b}$

if solution $\mathbf{v}$ is not admissible

then assign flow vector estimated at next coarser scale $j+1$ to this grid position

else assign current solution $\mathbf{v}$ to this grid position end for

Step 3: if $j>j_{\min }$ then go to finer scale: $j:=j-1$; goto Step 2

A solution of a local linear system is regarded as not admissible if the linear system is either ill-conditioned or the length of the central motion vector exceeds some scale-dependent limit. If the time derivative at a given location is below a pre-defined noise level, the local motion estimate is set to zero.

\section{Numerical Results}

The performance of the algorithm was tested on synthetic and real image sequences. In particular, we used the well known synthetic sequence "Yosemite". Since the exact motion field is known, the error of the estimated motion field was computed using the angular error measure as defined in [11]. As real data we used the "Rubik Cube" sequence ${ }^{1}$. One frame of each sequence and its corresponding estimated motion field is shown in Figures 3 and 4. All sequences were prefiltered with a Binomial filter of variance $\sigma^{2}=1.5$. The angular error of the "Yosemite" sequence is $6.33^{\circ} \pm 9.98^{\circ}$ with a flow field density of $100 \%$. The error of the corresponding adaptation of the Lucas-Kanade approach (same window, same multiresolution strategy) is $7.43^{\circ} \pm 12.72^{\circ}$. The rotational movement in the "Rubik Cube" sequence is also clearly recovered. These results compare favorably with all methods evaluated in the survey of Barron \& al. [11].

\section{REFERENCES}

[1] Ming-Kuei Hu, "Visual pattern recognition by moment invariants," IRE Trans. on Inf. Theory, pp. 179-187, 1962.

${ }^{1}$ All sequences were downloaded from Barron \& al.'s FTP site at ftp://csd.uwo.ca/pub/vision.
Fig. 4. One frame of the Rubik Cube sequence and its corresponding estimated motion field.

[2] C.H. Teh and R.T. Chin, "On image analysis by the methods of moments," PAMI, vol. 10, no. 4, pp. 496-513, 1988.

[3] S.A. Dudani, K.J. Breeding, and R.B. McGhee, "Aircraft identification by moment invariants," IEEE Trans. on Computers, vol. 26, no. 1, pp. 39-46, 1977.

[4] B.C. Li and J. Shen, "Fast calculation of local moments and application to range segmentation," Proc. Int. Conf. on Pattern Recognition, The Haage, vol. C, pp. 298-301, 1992.

[5] Q. Gao and F.F. Yin, "Two-dimensional direction-based interpolation with local centered moments," Graphical Models and Image Processing, vol. 61, no. 6, pp. 323-339, 1999.

[6] B. Lucas and T. Kanade, "An iterative image restoration technique with an application to stereo vision," Proc. of the DARPA IU Workshop, pp. 121-130, 1981.

[7] M.J. Shensa, "The discrete wavelet transform: wedding the à trous and mallat algorithms," IEEE Trans. on Signal Processing, vol. 40, no. 10, pp. 2464 - 2482, 1992.

[8] M. Unser, "Splines: A perfect fit for signal and image processing," IEEE Signal Processing Magazine, vol. 16, no. 6, pp. 22-38, 1999.

[9] J. Dubochet, M. Adrian, J.J. Chang, J.C. Homo, J. Lepault, A.W. McDowall, and P. Schultz, "Cryo-electron microscopy of vitrified specimens," Quart. Rev. of Biophysics, vol. 21, no. 2, pp. 129-228, 1988.

[10] B. Horn and B. Schunk, "Determining optical flow," Artificial Intelligence, vol. 17, pp. 185-203, 1981.

[11] J. Barron, D. Fleet, S. Beauchemin, and T. Burkitt, "Performance of optical flow techniques," IJCV, vol. 12, pp. 43-77, 1994. 